\title{
Pilocarpine on the Clinical Research in Treating Glaucoma
}

\author{
Yun Pan \\ Shijiazhuang Medical College \\ Chinese Hebei Shijiazhuang \\ panyunye123@163.com
}

\begin{abstract}
Glaucoma is one of the most common blinding eye disease, a serious threat to visual nerve function. Incidence may have from the freshmen do old people, and not easily noticed. However, the pathogenesis of glaucoma is not very clear. If not taking timely and effective treatment of glaucoma, vision will gradually reduce and even complete loss. So for early detection, early diagnosis and early treatment of glaucoma, Jaborandi alkali were introduced in this paper for the physical and chemical properties, pharmacologica mechanisms and clinical applications. Focusing on the pilocarpine clinical side effects in lowering intraocular pressure, intraocular pressure, effect on ocular blood flow and the application of visual function protection.
\end{abstract}

Keywords- western medicine; pilocarpine; clinical application

\section{INTRODUCTION}

At present, the incidence of glaucoma in increased rapidly, mostly in Asia, especially in China, yellow was the most common, the rare black, white, in more than 40 years old, the prevalence peak between 55-75 years of age, female, female incidence rate was 3-4 times higher than that of males, patients there are many hypermetropia. So for our country to further study the glaucoma, improve the early treatment of imminent.

\section{CLINICAL STUDY OF PILOCARPINE}

\section{(MATERIALS AND METHODS)}

\section{A. Long-term application of pilocarpine in rabbit corneal endothelial cells have no effect and influence.}

Methods: $2 \%$ pilocarpine in rabbit eye drops for 1 months and 3 months later, remove the rabbit eye quickly fixed, separation of corneal endothelial tissue, with Western blot (Western? Blot) anti apoptosis gene BCL method for detection of rabbit corneal endothelial cells and the expression of Bax? 2 protein expression, detection of corneal endothelial cell apoptosis by flow cytometry, ultrastructural changes of the corneal endothelial cells were observed under transmission electron microscope. Results: the application of pilocarpine in 1 months and 3 months later, showed the apoptosis of endothelial cells of rabbit corneal gene Bax protein expression increased, the anti-apoptotic gene BCL? 2 protein expression decreased, 3 month group of rabbit corneal endothelial cell apoptosis rate was higher than the control group $(\mathrm{P}<0.05)$, transmission electron microscope observation shows, 1 and 3 months group of intracellular vacuolization, mitochondrial swelling, dilatation of endoplasmic reticulum, nuclear pyknosis, chromatin pyknosis surrounding the typical changes of apoptosis. Conclusion: long-term application of $2 \%$ pilocarpine can induce the apoptosis of corneal endothelium cells in rabbits.

\section{B. Miotic effect comparison of 1\% pilocarpine liposome eye} drops and $1 \%$ pilocarpine eyedrops on the rabbit eyes.

Methods: 18 rabbits, randomly divided into experimental group, positive control group, negative control group. The experimental group of $1 \%$ pilocarpine liposome eye drops, the positive control group 1\% pilocarpine eye drops, the negative control group application blank liposome eye drops. All rabbits left eye drop in eye drops, eye drops of human physiological saline as control, before administration. After administration of $0.25,0.5,1,2,3,4,5,7 \mathrm{~h}$ measurement of pupil diameter. Results: the 3 groups before dropping the pupil diameter around eyes, no significant difference $(\mathrm{P}>0.05)$. After instillation of $0.25 \mathrm{~h}$, positive control group $(\mathrm{P}<0.01)$, the largest miotic effect drops $1 \mathrm{H}$ pupil began to disperse, differences in $5 \mathrm{~h}$ were not significant $(\mathrm{P}>0.05)$; the experimental group after the eye drops $0.5 \mathrm{~h}$ pupil minimum $(\mathrm{P}<0.01)$, 3h pupil began to disperse, $7 \mathrm{~h}$ had no significant difference $(\mathrm{P}>0.05)$; negative as observed in the $7 \mathrm{~h}$ group had no difference $(\mathrm{P}>0.05)$. Conclusion: $1 \%$ pilocarpine liposome eye drops with slow release, prolong the action time advantages.

\section{Comparative study on the pharmacokinetics of $2 \%$ pilocarpine microemulsion eye drops and $2 \%$ pilocarpine eye drops in aqueous humor of rabbit.}

Methods: 60 white rabbits, were randomly divided into 20 groups, 10 experimental groups, 10 control groups, each group of 3 rabbits (6 eyes). The experimental group eyes conjunctival sac accurately drip $2 \%$ pilocarpine microemulsion eye drops of $50 \mu \mathrm{L}$, the control group with the method of eye drops into the $2 \%$ pilocarpine eye drops of $50 \mu \mathrm{L}$, respectively after administration of 5, 10, 30, 40, 60, 90, 120, 180, 240 and 360 min extraction of real wate. Drug extraction with dichloromethane real water, by reversed phase high performance liquid chromatography (high pressure liquid chromatography, HPLC) method determination of aqueous concentration of drug. Results: the pilocarpine separation and other impurities by HPLC method, the minimum detectable concentration was $0.01 \mathrm{mg} / \mathrm{L}$. $2 \%$ pilocarpine microemulsion eye drops and eye drops eye drops after $50 \mu \mathrm{L}$, except at the time of $5 \mathrm{~min}$, the rest of the time points of pilocarpine microemulsion aqueous drug concentration eye treatment group compared with the pilocarpine eye drops in the treatment group were significantly increased $(\mathrm{P}=0.0003 \sim 0.042)$. Area of microemulsion eye drops under concentration-time curve is 3 
times of eye drops eye drops, microemulsion bioavailability than eye drops 2 times. Using microemulsion drops after $6 \mathrm{~h}$, the concentration of real water can still be detected with pilocarpine eye drops, and after $3 \mathrm{~h}$, the real water has not measured concentration of pilocarpine. Conclusion: pilocarpine eye drops form into microemulsion eye drops can significantly improve the pilocarpine bioavailability, enhance the efficacy, reduce drug use frequency, improve the treatment of glaucoma index, has better application prospect.

D. $1 \%$ pilocarpine liposome eye drops on the curative effect and adverse reaction of primary angle closure glaucoma.

Methods: primary angle-closure glaucoma in 60 eyes of 60 cases, including 30 cases in the control group, 30 cases; 30 cases of test group, 30 eyes. Before and after treatment, intraocular pressure and pupil diameter measurement, inspection of the real angle and the visual acuity, corneal, conjunctival, anterior chamber, iris, pupil, lens, fundus, A type ultrasonic measurement of anterior chamber depth, thickness of lens, ocular axis, at the same time recording in patients with symptoms of.1\% pilocarpine eye drops (control group): each eye drops 1 drops, about $50 \mu \mathrm{L}, 1 \mathrm{D} 4$; Of $1 \%$ pilocarpine liposome eye drops (test group): each eye drops 1 drops, about $50 \mu \mathrm{L}, 1 \mathrm{~d} 2$. Results of the 2 groups in sex, age, before treatment, pupil diameter intraocular pressure by statistical test pressure $\mathrm{P}>0.05$, there was no significant difference between the patients of group.2 after instillation time decreased compared with before treatment, the difference was significant (paired t test, $\mathrm{P}<0.05$ ); comparing drops each time after the 2 groups of patients with hypertension intraocular pressure, test group decreased significantly, there was significant difference (independent samples $t$ test, $\mathrm{P}<0.05$ ) patients in group. 2 drops each time after the pupil value compared with before treatment, the pupil decreased, there was a significant difference (paired $t$ test, $\mathrm{P}<0.05$ ); Drop pills each time after 2 groups of pupil values compared to experimental group, the pupil is small, there was a significant difference (independent samples $t$ test, $\mathrm{P}<0.05)$. Each group before and after treatment in 2 groups of patients before and after medication and anterior chamber depth, thickness of lens and eye axial length, the statistical test, no difference. Liposome group there were 7 cases of surface sting occurs after instillation of 2 short, no adverse reaction was found in other ocular and systemic. Conclusion: the treatment of pilocarpine liposome eye drops used in primary angleclosure glaucoma, can reduce eye drop frequency, effective control of intraocular pressure.

\section{PHARMACOLOGICAL ACTION OF PILOCARPINE}

\section{THE ROLE OF EYE}

(1) The pupil iris sphincter: excited on the M cholinergic receptor, the iris sphincter contraction.(2)To reduce intraocular pressure: through the action of miosis, the iris to iris root central tension, thinning, the anterior chamber angle gap expansion, real water easily through the trabecular meshwork and episcleral veins sinus into the cycle, reduce intraocular pressure. The accommodative spasm: excited $\mathrm{M}$ receptors on the ciliary muscle, the ciliary muscle contraction to the pupil center direction, suspensory ligament relaxation, lens by itself is elastic and variable convex, diopter increasing, so as far as near objects clearly, blurred.

\section{CLINICAL APPLICATION OF JABORANDI ALKALI}

\section{A. Human application}

In humans, the main clinical locally for the treatment of glaucoma. After the eye drops can easily pass through the cornea into the eye, decreased intraocular pressure, occurring after 10 minutes, half an hour, it can alleviate or eliminate glaucoma due to high intraocular pressure, headache, visual loss and other symptoms. Compared with physostigmine, pilocarpine effect of mild and transient, so the dosing interval should be short, relatively stable water solution. Absorption after the adverse reaction is mainly composed of the $M$ cholinergic effect caused by atropine, available against. Glaucoma, increased intraocular pressure is the main feature of glaucoma, can cause headache, visual loss and other symptoms, serious when can cause blindness. Angle-closure glaucoma (acute or chronic congestive glaucoma) in patients with stenosis of anterior chamber angle, increased intraocular pressure. Pilocarpine can make the intraocular pressure decreases rapidly, so as to eliminate or relieve the symptoms of glaucoma. Pilocarpine is also suitable for open angle glaucoma (chronic simple glaucoma) treatment. The anterior chamber angle glaucoma without stenosis and occlusion, but because of the trabecular meshwork and sinus venosus sclerae denaturation or hardening, hampering the real water circulation, causing elevated intraocular pressure. Pilocarpine may by surrounding the small blood vessels dilatation and contraction of the ciliary scleral venous sinus, trabecular meshwork structure is changed and the intraocular pressure drop. This drug can be used for the neck after oral dry mouth, but the increase in the secretion of saliva, sweat secretion increased significantly.

\section{B. clinical research}

- 1)To understand the curative effect and adverse reaction of domestic pilocarpine treatment of xerostomia induced by antipsychotics.

- Method: dry mouth caused by psychotropic drugs in 75 cases, 60 patients were divided into two groups, each group of male and female patients in 15 cases, double blind randomized control method were given pilocarpine $(2.5 \mathrm{mg} /)$ or placebo (starch) the same shape 2 tablets, oral TID, for $28 \mathrm{~d}$. The other 15 patients the same method to pilocarpine open treatment. Results: pilocarpine, the total effective rate was $77.8 \%$ in the placebo group, the natural rate was $26.7 \%$; comparison between groups, $\mathrm{P}<0.01$. Adverse reactions include sinus bradycardia, sweat, tears and blurred vision. Conclusion: the drug safe and effective for dry mouth caused by psychotropic drugs.

- 2) Study of $1 \%$ pilocarpine liposome eye drops on the curative effect and adverse reaction of primary angleclosure glaucoma.

- Methods: primary angle-closure glaucoma in 60 eyes of 60 cases, including 30 cases in the control group, 30 cases; 30 cases of test group, 30 eyes. Before and after treatment, intraocular pressure and pupil diameter 
measurement, inspection of the real angle and the visual acuity, corneal, conjunctival, anterior chamber, iris, pupil, lens, fundus, A type ultrasonic measurement of anterior chamber depth, thickness of lens, ocular axis, at the same time recording in patients with symptoms of.1\% pilocarpine eye drops (control group): each eye drops 1 drops, about $50 \mu \mathrm{L}, 1 \mathrm{D} 4$; Of $1 \%$ pilocarpine liposome eye drops (test group): each eye drops 1 drops, about $50 \mu \mathrm{L}, 1 \mathrm{D} 2$. The results of 2 groups of patients gender, age, before treatment, the diameter of the pupil was tested by statistics $\mathrm{P}>0.05$, without significant difference. Intraocular pressure in 12 groups of patients after instillation time decreased compared with before treatment, the difference was significant (paired t test, $\mathrm{P}<0.05$ ); comparing drops each time after 2 groups of patients with intraocular pressure, intraocular pressure decreased significantly in experimental group, there was significant difference (independent samples $t$ test, $\mathrm{P}<0.05$ ) patients in group. 2 drops after each time the pupil value compared with before treatment, the pupil decreased, there was a significant difference (paired t test, $\mathrm{P}<0.05$ ); Drop pills each time after 2 groups of pupil values compared to experimental group, the pupil is small, there was a significant difference (independent samples $\mathrm{t}$ test, $\mathrm{P}<0.05)$. Each group before and after treatment in 2 groups of patients before and after medication and anterior chamber depth, thickness of lens and eye axial length, the statistical test, no difference. Liposome group there were 7 cases of surface sting occurs after instillation of 2 short, no adverse reaction was found in other ocular and systemic. Conclusion: the treatment of pilocarpine liposome eye drops used in primary angleclosure glaucoma, can reduce eye drop frequency, effective control of intraocular pressure.

\section{ACKNOWLEDGMENT}

Now glaucoma incidence increased, although there are some genetic factors, but more important is the impact of external factors, eyesight burden. Prevention focus is to develop good habits with the eye, but also should begin from childhood. This not only can progress of myopia and myopia prevention, can also occur in glaucoma and other eye. Once appear due to high intraocular pressure caused by headache, visual loss and other symptoms, should be early in the prevention, early availability of pilocarpine medication under the guidance of a doctor's treatment, reduce intraocular pressure to ensure the normal circulation of intraocular travel body, maintain the optical properties of refractive media, the visual is very important function.

\section{REFERENCES}

[1] People's Medical Publishing House " The eye disease study ". Editor: Zhu Renyuan

[2] Higher education press " The ophthalmology " editor in chief: $\mathrm{Xu}$ Guoxing

[3] People's Medical Publishing House "pharmacology and therapeutics based" edited by Zhang Qing

[4] Medical education net

[5] Fitzgerald P, Hadgraft J, Wilson CG. A gamma scintigraphic evaluation of the precorneal residence of liposomal formulations in the rabbit. J Pharm pharmacol, 1987,39:487-490.

[6] Naveh N, Muchtar S, Benita S. Pilocarpine incorporated into a submicron emulsion vehicle cause an unexpectedly prolonged ocular hypotensive effect in rabbits. J Ocular Pharmacol, 1994,10:509-520.

[7] Beilin M, Bar-Ilan A, Amselem S,et al. Ocular retention time of submicron emulsion (SME) and the miotic response to pilocarpine delivered in SME. Invest Ophthalmol Vis Sci (ARVO), 1995,36:s166.

[8] Mahato RI, Thies C, Lubniewski A,et al. Polymer mixture nanoparticle for ocular drug delivery. Invest Ophthalmol Vis Sci (ARVO), 1994,35:1387.

[9] Muchtar S, Almog S, Torracca MI, et al. A submicron emulsion as ocular vehicle for delta-8-tetrahydrocannabinol:effcct on intraocular pressure in rabbits. Ophthalmic Res,1992,24:142-149.

[10] Wang Hongxi, Jiang Xuetao. Research progress of microemulsion. Foreign Med sci,1996, 23 : 206-211

[11] Yang Baofeng. The pharmacology (Seventh Edition): People's Medical Publishing House, 2011:61 